\title{
Ubicuidad en sistemas de telecomunicaciones a través de laboratorios virtuales ${ }^{1}$
}

\author{
Gómez R. Jorge ${ }^{2}$ Camargo A. Leonardo $\quad$ Medina D. Byron ${ }^{4}$
}

Recibido:

Agosto 2 de 2012

Aceptado:

Noviembre 28 de 2012
${ }^{1} \mathrm{El}$ artículo es el resultado de un trabajo de desarrollo de grupos de investigación, con el fin de implementar estrategias de aprendizaje con nuevas tecnologías de información .

${ }^{2}$ Magíster en Ingeniería Electrónica, Grupo de Investigación en Desarrollo Electrónico y Aplicaciones Móviles GIDEAM, Universidad del Magdalena, Carrera 32 No. 22-08 Sector San Pedro Alejandrino, Santa Marta, Colombia, Correo electrónico:

jgomez@unimagdalena.edu.co

${ }^{3}$ Magíster en Ingeniería Electrónica, Grupo de Investigación en Desarrollo Electrónico y Aplicaciones Móviles GIDEAM, Universidad del Magdalena, Carrera 32

No. 22-08 Sector San Pedro Alejandrino, Santa Marta, Colombia, Correo electrónico: lcamargoa@unimagdalena.edu.co

Magíster en Ingeniería Electrónica, Grupo de Investigación y Desarrollo en Telecomunicaciones GIDT, Universidad Francisco de Paula Santander, Avenida Gran Colombia No. 12E-96B Colsag, Cúcuta, Colombia, Correo electrónico: bmdelgad@ufps.edu.co

\section{Resumen}

Basados en la tecnología y el auge de los sistemas de información y telecomunicaciones se presenta un aplicación para atender la necesidad a la optimización de recursos en los programas académicos de ingeniería electrónica. Utilizando técnicas para la virtualización de recursos educativos, se desarrolló una plataforma virtual para el adiestramiento y entrenamiento ubicuo en el área de las telecomunicaciones "Labsistel", la realización de este proyecto contribuye con el desarrollo académico de los estudiantes de ingeniería electrónica, brindándoles una herramienta que le facilita a cualquier programa de ingeniería electrónica abarcar la temática de las cátedras, aprovechando los espacios, con el fin de enriquecer los conocimientos.

Palabras clave: TIC, sistema de telecomunicaciones, gestor de contenido.

\section{Abstract}

Based on technology and the rise of information and telecommunications systems, we present an application to address the need to optimize resources for the academic program of electronics engineering. We created a virtual platform called "Labsistel" for the ubiquitous training of students in the area of telecommunications. We used techniques for the virtualization of the educational resources. This project contributes to the academic development of the electronics engineering students, providing a tool that allows any electronics engineering program to cover the content of the lectures, using new technologies to improve the learning experience.

Keywords: TIC, Telecommunications System, Content Manager. 


\section{Introducción}

$E^{1}$ 1 progreso de las comunicaciones unido a la evolución de herramientas procesos informáticos, han transformado la manera de compartir la información y las ideas, generando cambios trascendentales en los sistemas educativos actuales, principalmente en la educación superior [1]. Este surgimiento ha permitido en los últimos años incursionar enormemente en los procesos de formación profesional de la educación superior. El crecimiento exponencial de estas tecnologías permitió el avance de nuevos software como método de enseñanza, utilizados principalmente en la elaboración de laboratorios específicos. Éstos son cada día más potentes y con la capacidad de simulación interactiva, las cuales han contribuido a desarrollar habilidades cognitivas en el estudiante [2].

La virtualidad es la forma de emplear un recurso sin disponer de él físicamente. A través de las Tecnologías de la Información y Comunicaciones (TIC) es posible conseguir ubicuidad, y sinérgicamente con la virtualidad se potencializan herramientas para el desarrollo de competencias y en sí para desarrollar recursos virtuales de aprendizaje. Desde el surgimiento del concepto de laboratorio virtual, el modelo de educación ha experimentado transformaciones en las distintas técnicas de enseñanza [3], de tal forma que las asignaturas sean evaluadas en sus propios términos [4]. La utilización de este medio hoy en día juega un papel importante en la educación superior, gracias a las ventajas de este tipo de tecnología como base en los nuevos modelos de enseñanza, tanto activo como colaborativo; con el fin de permitirle a los estudiantes suplir sus necesidades educativas [1].

Desde el punto de vista de la enseñanza de las telecomunicaciones, la realización de prácticas experimentales sobre sistemas reales es de suma importancia ya que, no solamente ayuda a los estudiantes a asimilar los conceptos teóricos; sino que también, desvela otros aspectos difíciles de abordar de forma teórica. Este tipo de enseñanza genera una necesidad insaciable de conocimiento y representa desafíos importantes en los procesos de educación, entrenamiento, actualización y mejoramiento de habilidades [5].

En las universidades acreditadas de alta calidad, específicamente el programa de Ingeniería Electrónica, cuentan con software licenciado, que cumple con ciertos términos de uso, establecidos por el fabricante. Estas herramientas son consideradas herramientas institucionales usadas en las cátedras del programa, que al mismo tiempo son fundamentales para el progreso académico [6].

Así mismo los programas académicos tienen establecidos espacios para las prácticas de laboratorios, que complementan las asignaturas de cada área de formación profesional; fundamentalmente en los sistemas de telecomunicaciones. Algunos programas no cuentan con las licencias suficientes ni la disponibilidad de dispositivos, guías o manuales de ejecución de las prácticas de laboratorios adecuados para suplir las experiencias de las temáticas teóricas concernientes a los sistemas de comunicación.

La tendencia global en países de avanzada es que los programas de pregrado se enfoquen en herramientas de simulación; más que en la misma implementación, debido a que éstas permiten que se desarrollen habilidades en el análisis, el diseño y la síntesis, con el fin de entender el sistema [7]. La simulación de los laboratorios tiene como finalidad modelar y variar parámetros para analizar sus cambios o alteraciones, afines a las condiciones de diseño, para poder comprobar los resultados obtenidos y así proseguir a la implementación.

El tiempojuega un papel muy importante en el desarrollo de las temáticas de las asignaturas correspondientes a las comunicaciones, debido a que se necesitan horas teóricas, las cuales son asistidas por el docente; horas 
prácticas, en las cuales se llevan a cabo las experiencias en los laboratorios y, horas autónomas empleadas por los estudiantes para desarrollar las actividades programadas [8]. El diseño de los laboratorios virtuales le permite al discente volverse gestor de su propio aprendizaje y al profesor convertirse en facilitador, colaborador y orientador de ese proceso [9], es decir, potencializar el trabajo independiente en sus horas autónomas.

Por medio de este proyecto se buscó realizar aprendizajes autónomos basados en experiencias de laboratorios en las diferentes áreas de las telecomunicaciones, en el que a través de aplicaciones para computadoras se pueda simular las temáticas fundamentales para el desarrollo de la asignatura y a su vez reforzar la teoría, permitiendo al estudiante una mejor demostración de los conceptos tratados en las horas de clases.

Por medio del laboratorio virtual se logra ampliar el espectro de ocupación de los estudiantes, permitiendo a los alumnos realizar sus prácticas sin restricciones de horario y con la posibilidad de ejecución ilimitada.

El diseño de los laboratorios virtuales, fortalece los cursos en el área de las telecomunicaciones y le permite a las universidades dinamizar y explotar al máximo los recursos de los laboratorios tangibles empleados por el programa de Ingeniería Electrónica para dedicarlos a la investigación.

\section{Materiales y métodos}

La metodología utilizada para desarrollar el proyecto es la de Estructura de Descomposición del Trabajo o EDT, también conocida por su nombre en inglés Work Breakdown Structure o WBS, es en gestión de proyectos una descomposición jerárquica orientada al entregable, del trabajo a ser ejecutado por el equipo del proyecto, para cumplir con los objetivos de éste y crear los entregables requeridos, con cada nivel descendente de la EDT, representando una definición con un detalle incrementado del trabajo del proyecto. La EDT es una herramienta fundamental en la gestión de proyectos [10].

El propósito de una EDT es organizar y definir el alcance total aprobado del proyecto según lo declarado en la documentación vigente. Su forma jerárquica permite una fácil identificación de los elementos finales, llamados paquetes de trabajo. Se trata de un elemento exhaustivo en cuanto al alcance del proyecto, la EDT sirve como la base para la planificación del proyecto. Todo trabajo a realizar en el proyecto debe poder rastrear su origen en una o más entradas de la EDT [11].

Es por todo lo anterior que se utiliza esta metodología para el desarrollo del proyecto, permitiendo la libertad de dividir de manera flexible los trabajos y las tareas a realizar, en donde los objetivos se distribuyen en actividades y, las actividades se fraccionan en tareas.

\section{Fase I: Determinar las temáticas.} ISSN 0122-820X

- Recopilación de datos: se realizó la documentación referente a las temáticas impartidas en el área de las telecomunicaciones.

- Análisis de la información: se clasificó por orden secuencial según el diseño curricular vigente, la información relevante obtenida en la actividad anterior y se determinaron las temáticas de amplitud modulada, frecuencia modulada, televisión analógica, muestreo, codificación de línea y modulación digital.

\section{Fase II: Diseño de una plataforma} interactiva y dinámica.

- Análisis de herramientas: se realizó un estudio de los diferentes software que cumplen con las características para el desarrollo del proyecto. Los criterios de selección son el tipo de licenciamiento, las potencialidades gráficas, la actualización en línea de la plataforma, la seguridad 
y, la usabilidad. Se determina que los software Joomla, Hot Potatoes, LabView y Matlab cumplen con las características del proyecto.

- Análisis funcional del software: se inicia la etapa de conocimiento e interacción con el software, en aras de garantizar la eficiencia del mismo en el proceso de estructuración de las etapas.

- Diseño de las etapas: se plantean las diferentes etapas que va a tener la plataforma virtual y el diseño respectivo al modelo metodológico que más se adecúa a la representación de cada etapa (ver figura 1)

En la etapa de iniciar sesión el estudiante y/o el docente se validan e ingresan a la plataforma.

Figura 1. Etapas de los laboratorios virtuales.

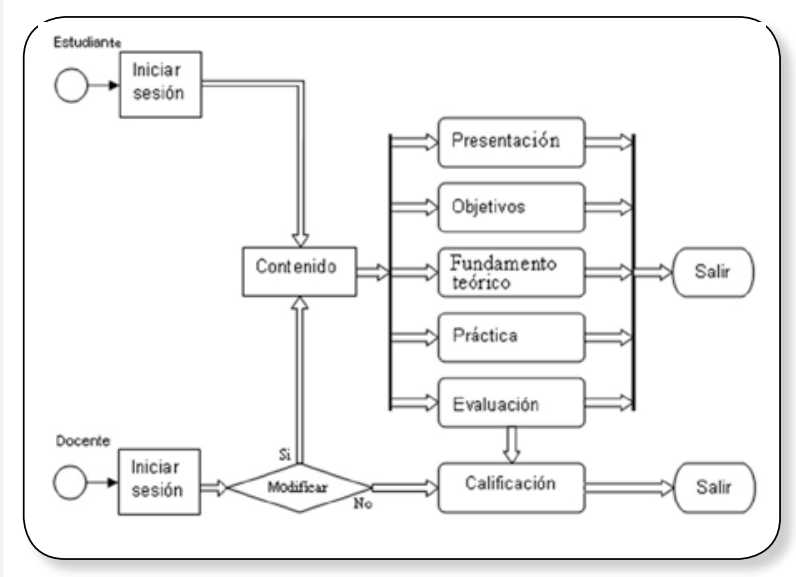

En la etapa de contenido el docente crea y modifica el material que el estudiante observa y realizará en su laboratorio. El contenido de la plataforma está subdividido en presentación, objetivos, fundamento teórico, práctica y evaluación.

En la presentación el estudiante y/o el docente encuentran una pequeña introducción de la temática.

En los objetivos se determinan las destrezas y habilidades a adquirir y desarrollar a cerca de la temática.
El fundamento teórico representa los conceptos relevantes de la temática y su aplicación en la vida real antes de realizar las prácticas.

En la práctica se ejercitan los conocimientos adquiridos, asociando los términos teóricos asimilados durante la interacción con la plataforma.

En la evaluación el estudiante mide los conocimientos adquiridos con respecto a la temática, en una prueba corta. Las preguntas se toman aleatoriamente de una base de datos que se encuentra en el servidor donde se aloja el aplicativo. Del mismo modo, esta base de datos es actualizable por el docente con su respectiva autenticación. Solo se permite el paso de una temática a otra con la aprobación de la evaluación.

En la etapa de calificación se asigna un valor numérico al trabajo realizado por el estudiante dentro de la plataforma.

\section{Fase III: Implementación de laboratorios virtuales.}

- De acuerdo con los diseños desarrollados en la fase anterior, se procede a la construcción de los algoritmos correspondientes a cada laboratorio, pero cada uno con su respectiva interfaz gráfica; posteriormente, se realiza la integración de los laboratorios.

\section{Fase IV: Verificación.}

- Se realizan pruebas a la plataforma virtual, para determinar el nivel de confianza y de facilidad, al utilizar los laboratorios virtuales, permitiendo establecer ajustes en los diseños elaborados en las fases anteriores. En la figura 2 se muestra la interfaz de presentación de la herramienta, en una práctica aplicada de modulación en amplitud que es la temática central de un curso de esta índole. 
Figura 2. Práctica virtual acerca de modulación en amplitud desde LABSITEL.

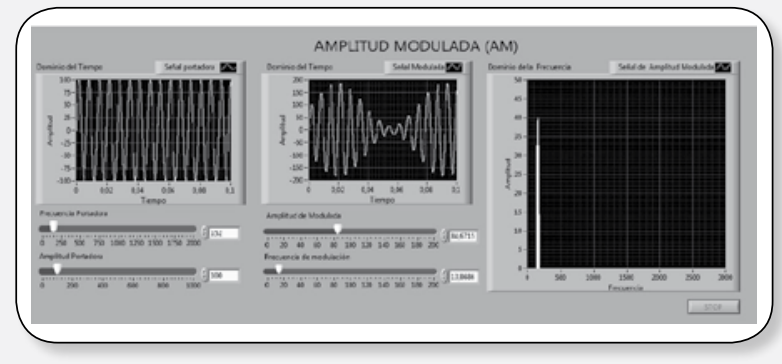

\section{Desarrollo de Labsistel}

\section{- Arquitectura de Labsistel}

La plataforma utiliza una arquitectura cliente-servidor, que permite al cliente interactuar con el sistema en un ambiente distribuido. La información de registro, acceso y evaluación, es almacenada en una base de datos que se puede acceder a través de Internet, requiriendo que el cliente y el servidor manejen documentos Web.

Se emplea MATLAB y LabView como herramientas de procesamiento matemático y procesamiento de señales, respectivamente. Estos son software licenciados que se emplean en la mayoría de las instituciones acreditadas con calidad y que poseen una Facultad de Ingeniería.

\section{- Funcionalidades}

La interfaz de usuario (ver figura 3) del sistema está dividida en dos partes, una pública y otra privada. En la parte pública, el estudiante sólo puede iniciar sesión. En la parte privada sólo se tiene acceso si se está registrado, en ésta, el usuario interactúa con todos los elementos del sistema y, desarrolla las prácticas y evaluaciones de las temáticas.

En la opción de Ingreso se presenta un formulario en una página PHP, donde el usuario se registra para ingresar a la parte privada de la plataforma. La plataforma verifica que el usuario esté registrado en la base de datos, en caso contrario, el sistema niega el acceso a éste y le indica que sólo puede hacer uso de la plataforma realizando el respectivo registro. Luego de comprobar la existencia del usuario en la base de datos, un servlet se comunica con el servidor, permitiendo el acceso al entorno de la plataforma.

Figura 3. Esquema de las interfaces de la plataforma.

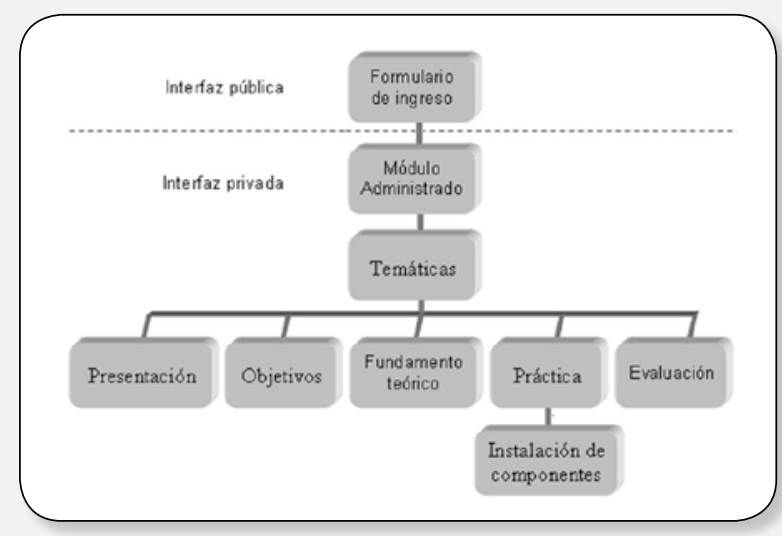

En Labsistel se emplearon documentos estáticos, dinámicos y activos.

La información contenida en los documentos Web estáticos reside en el servidor y, cada vez que se hace una solicitud a documentos de este tipo, se entrega siempre la misma respuesta, como ejemplo, las presentaciones, objetivos y fundamento teórico de cada temática.

Los documentos Web dinámicos son creados por el servidor como respuesta a la solicitud de un usuario, por lo que no existen de forma predeterminada en el servidor. Estos documentos presentan un comportamiento dinámico debido, principalmente, a que son controlados por un programa de aplicación. Cuando la solicitud del cliente llega al servidor, éste ejecuta un programa de aplicación que crea el documento y lo devuelve al cliente. Debido a que con cada nueva solicitud se crea un nuevo documento, el contenido del documento es dinámico y, presenta variaciones entre una y otra consulta, como por ejemplo las evaluaciones aplicadas en Labsistel. ISSN 0122-820X 
lado del cliente. Si un documento de este tipo es solicitado por el cliente, el servidor hace la gestión y devuelve al usuario la copia de un programa que se ejecutará localmente. El programa del documento activo permite la interacción directa con el usuario, y éste último, puede manipularlo para alterar constantemente el contenido de la información que se presenta, como por ejemplo las prácticas de laboratorios.

\section{Evaluación}

Dado que la herramienta elaborada está orientada a desarrollar las competencias básicas de la observación, la comparación y el contraste, Labsistel tiene una serie de datos almacenados que pueden ser modificados por los docentes, esto incluye la parte de la calificación. Por tal motivo, se somete a verificación la herramienta desde dos puntos de vista, el académico y el de software. Desde el plano académico es apreciable como el apoyo obtenido con Labsistel permitió a los estudiantes de un curso específico mejorar sus resultados frente al historial promedio. Desde el punto de vista de la ingeniería de software, la evaluación es un elemento crítico para la garantía de calidad del software y, representa una revisión final de las especificaciones, del diseño y de la codificación [12]. Una vez que se ha creado un prototipo de interfaz que funcione, debe evaluarse para determinar si satisface las necesidades del usuario. El espectro de evaluación puede ir desde una ejecución de prueba informal, en la que el usuario proporcione sus sensaciones, hasta un estudio diseñado formalmente que use métodos estadísticos para la evaluación de cuestionarios aplicados a una población de usuarios finales [12].

La evaluación de la interfaz se ha hecho utilizando la metodología para la evaluación de interfaces multimedia de Jakob Nielsen [13]. Aquí se desarrolla un concepto propio para medir y dar valor a las aplicaciones computarizadas. Dicho concepto es el grado de usabilidad que una aplicación alcanza [13].
Por definición, la palabra usabilidad puede ser confundida con utilidad, más este término sugiere un concepto más amplio. La usabilidad es la manera en que una aplicación computacional integra cualidades que la hacen eficiente, apropiada y económica, para los usuarios que la utilizan y para los equipos que la soportan [14]. La utilidad, se refiere más bien, a la factibilidad de uso en alguna área específica.

Mediante una evaluación de usabilidad es posible conocer cualidades del sistema que muestren el alcance al que puede llegar, al ser utilizado por usuarios específicos, para conseguir ciertas metas con eficiencia, efectividad y satisfacción, en un contexto de uso concreto.

Según Nielsen, el número de evaluaciones puede variar entre 1 y 15 . Nielsen muestra en una gráfica (ver figura 4), el número de problemas de usabilidad detectados en contraste al número de evaluadores que participan.

Como se puede observar en la figura 4, el número ideal de evaluadores según el estudio de Nielsen, debe encontrarse entre 3 y no más de 15 ó 20 participantes.

Figura 4. Proporción de problemas de usabilidad encontrados en la aplicación de una evaluación.

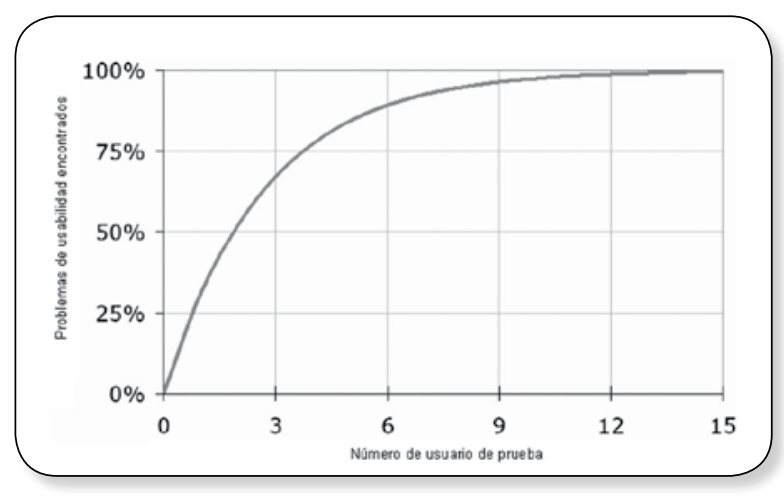

Fuente: http://www.useit.com/alertbox/20000319-usertesting-diminshing-returns-curve.gif

En el proyecto, se aplica el método propuesto por Nielsen para evaluar la interfaz en cuanto a su usabilidad, tomando en cuenta 
que debe reunir los siguientes atributos: la interfaz debe ser fácil de aprender, debe ser eficiente como herramienta de enseñanza, debe ser fácil de recordar, debe contener pocos errores y, debe ser satisfactoria y placentera.

En la tabla 1 se presenta el instrumento de evaluación aplicado a un grupo de estudiantes de Ingeniería Electrónica de la Universidad del Magdalena.

Tabla 1. Evaluación de usabilidad, Universidad del Magdalena.

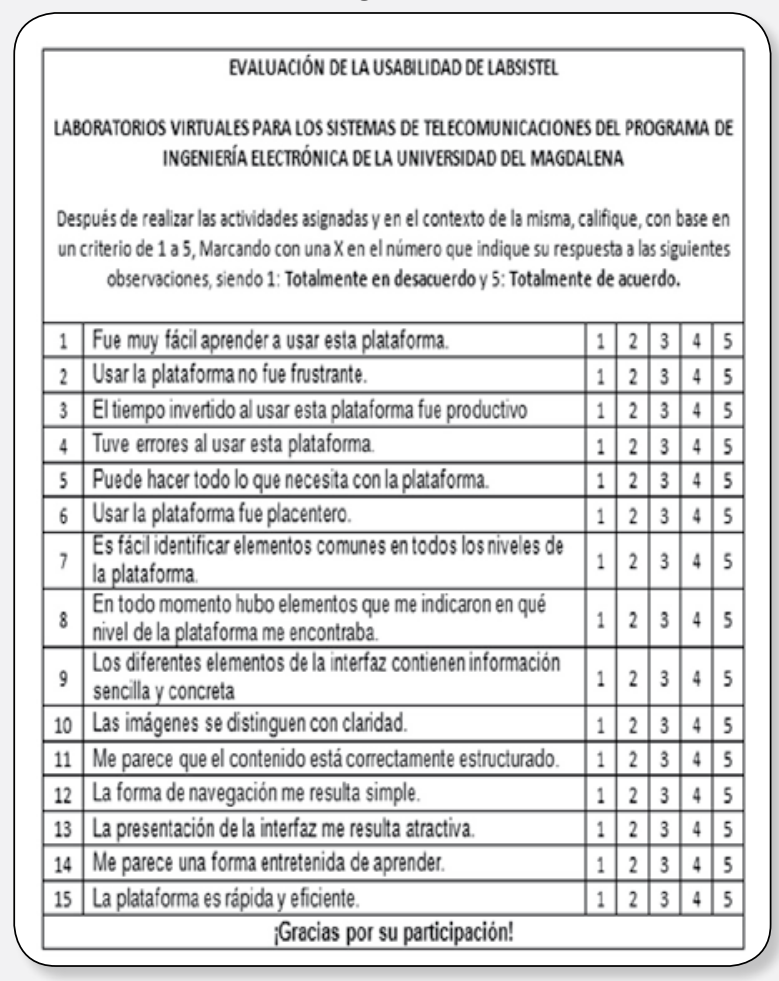

\section{Resultados}

En la evaluación del sistema se aplicaron 20 cuestionarios de evaluación; los resultados que se obtuvieron se aprecian en la tabla 2.

El grupo objetivo se conformó de alumnos de Ingeniería Electrónica de la Universidad del Magdalena, con conocimientos mínimos del uso de la computadora e Internet. Éstos, evaluaron la plataforma, haciendo uso de la misma durante un periodo de una (1) hora y, tuvieron diez (10) minutos para contestar el cuestionario de evaluación.
Diciembre 2012 ISSN 0122-820X

Tabla 2. Resultados de la evaluación de usabilidad de la interfaz.

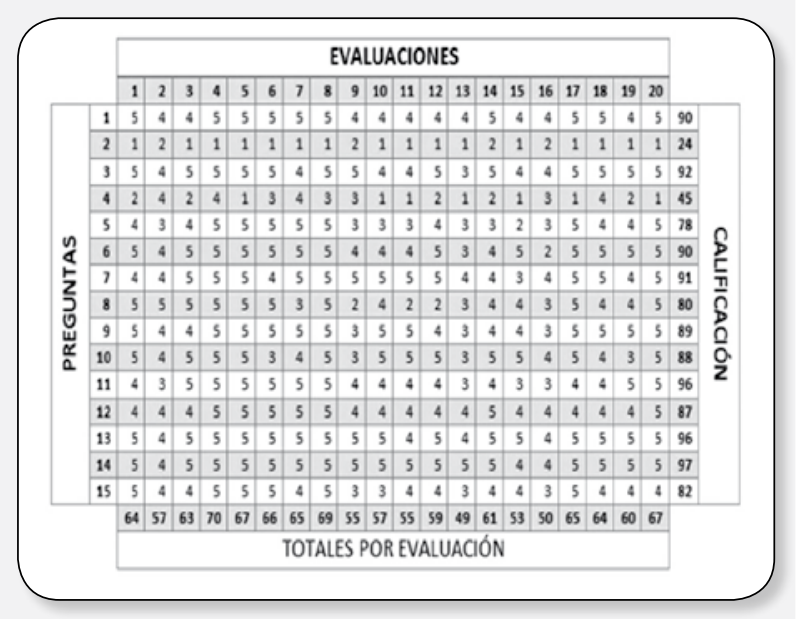

La escala de usabilidad establecida, se observa en la tabla 3, con el fin de evaluar por separado cada aspecto.

Tabla 3. Interpretación de los resultados.

\begin{tabular}{|c|c|l|}
\hline $\begin{array}{c}\text { ESCALA DE } \\
\text { USABILIDAD }\end{array}$ & RESULTADOS & $\begin{array}{l}\text { INTERPRETACIÓN DE } \\
\text { LOS RESULTADOS }\end{array}$ \\
\hline $20-36$ & $\begin{array}{c}\text { Catástrofe de } \\
\text { usabilidad }\end{array}$ & $\begin{array}{l}\text { Es imperativo resolver el } \\
\text { problema antes de liberar la } \\
\text { aplicación. }\end{array}$ \\
\hline $37-53$ & $\begin{array}{c}\text { Problema de } \\
\text { usabilidad mayor }\end{array}$ & $\begin{array}{l}\text { Es importante resolverlo con } \\
\text { alta prioridad. }\end{array}$ \\
\hline $54-70$ & $\begin{array}{c}\text { Problema de usabi- } \\
\text { lidad menor }\end{array}$ & $\begin{array}{l}\text { Se debe resolver, pero con } \\
\text { baja prioridad. }\end{array}$ \\
\hline $71-87$ & $\begin{array}{c}\text { Problema cosméti- } \\
\text { co solamente }\end{array}$ & $\begin{array}{l}\text { No es prioritario darle solu- } \\
\text { ción inmediata. }\end{array}$ \\
\hline $88-100$ & $\begin{array}{c}\text { No hay problema } \\
\text { de usabilidad }\end{array}$ & $\begin{array}{l}\text { No estoy de acuerdo en que } \\
\text { esto sea un problema de usa- } \\
\text { bilidad en lo absoluto. }\end{array}$ \\
\hline
\end{tabular}

La tendencia general puede observarse en la figura 5 .

Figura 5. Tendencias en aspecto de usabilidad de Labsistel.

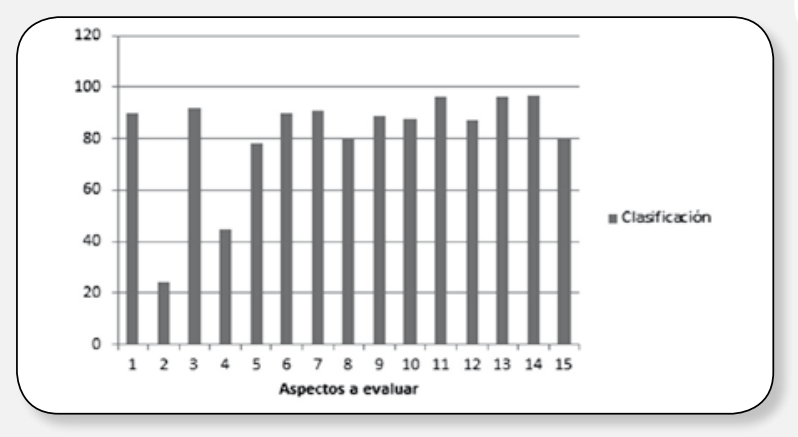


En general, se presentan aspectos favorables que indican la factibilidad de uso de la plataforma para aprender eficientemente sin que el discente detecte una gran cantidad de errores en el uso del sistema, ya que la mayoría de los estudiantes, lo considera atractivo y placentero según los resultados de la evaluación.

\section{Conclusiones}

Se obtiene una herramienta de educación virtual y ubicua que permite la integración del software licenciado. Esta estrategia va de la mano con la directiva del gobierno nacional de incrementar la cobertura de la educación superior empleando las TIC.

La metodología para la evaluación de interfaces multimedia de Jakob Nielsen, permitió evaluar la plataforma Labsistel en las diferentes etapas y en la totalidad, con la cual se pudo realizar una restructuración adecuada de la plataforma.

El diseño de las prácticas de laboratorios virtuales para los sistemas de telecomunicaciones aplicado al Programa de Ingeniería Electrónica de la Universidad del Magdalena contribuyó a la innovación y al desarrollo tecnológico, que orientan los procesos de laboratorios para el programa y, a su vez fortalecen los modelos de enseñanza tanto activo como colaborativo en las áreas de las telecomunicaciones.

El diseño de Labsistel facilita la comprensión de los conceptos aplicados en las temáticas principales de los sistemas de telecomunicaciones, porque desarrolla prácticas interactivas para los usuarios. La plataforma que se implementó, es un aporte al trabajo experimental al interior y al exterior de las aulas de clases, por medio de Internet.

\section{Referencias Bibliográficas}

[1] Eduardo Caicedo, Eval Bacca, Bayron Calvache, Jaiber Cardona y Jaime Buitrago, "Laboratorio distribuido con acceso remoto para la enseñanza de la robótica”. Educación en Ingeniería, volumen 4, número 7, Junio 2009, pp. 51-61.

[2] Ministerio de Educación Nacional, "Tecnología al Servicio de la Educación". Altablero, número 4, Mayo 2001, pp. 20.

[3] Rosado, L. y Herreros, R. J, "Nuevas aportaciones didácticas de los laboratorios virtuales y remotos en la enseñanza de la Física”. Formatex, 2005, pp. 1-2.

[4] Gustavo Meneses, César Ordosgoitia, "Laboratorio virtual basado en la metodología de aprendizaje basado en problemas, Abp". Educación en Ingeniería, volumen 4, número 7, Junio 2009, pp. 62-73.

[5] Julian Moreno, Demetrio Ovalle y Rosa Vicari, "Hacia una taxonomía en la educación asistida por computador". Educación en Ingeniería, volumen 5, número 9, Junio 2010, pp. 27-36.

[6] Decreto "Por el cual se modifica la estructura del Ministerio de Educación Nacional, y se determinan las funciones de sus dependencias". Decreto número 1306, 17 de Abril 2009.

[7] Jesús López y Mónica Delgado, "Laboratorio virtual de control inteligente". Educación en Ingeniería, volumen 4, número 8, Diciembre de 2009, pp. 102-110.

[8] Ministerio de Educación Nacional, "Sistema de Créditos Académicos". Altablero, número 10, Noviembre de 2001, pp. 1.

[9] Ministerio de Educación Nacional, "El Docente en un Nuevo Escenario". Educación Superior, número 4, marzo 2005, pp. 6. 
[10] Booz, Value Management Tutorial Module 2: Work Breakdown Structure, Mayo 6 2011, disponible en: http://science.energy.gov/ / media/opa/powerpoint/Final_Module_2.ppt

[11] NASA, NASA procedimientos y directrices, 6 de Mayo del 2011, disponible en: http://nodis3.gsfc.nasa.gov/displayCA.cfm?Internal_ ID $=$ N_PR_9501_002D_\&page_name $=$ Chp2

[12] Joaquín Fuentes, "Realidad Virtual Aplicada al Tratamiento del Trastorno de la lateralidad y Ubicación Espacial”, México, 2003.

[13] Jakob Nielsen y Robert L. Mack, Usability Inspection Methods. 1ㄹ edición, Jhon Wiley and Sons, Estados Unidos, 1994, pp. 41.

[14] Xavier Ferré Grau, "Principios Básicos de Usabilidad para Ingenieros de Software". Universidad Politécnica de Madrid, volumen 1, número 1, Mayo de 2010, pp. 1-8. 